\title{
Detection of lymphangiogenesis in non-small cell lung cancer and its prognostic value Jian-guo Sun ${ }^{\dagger 1}$, Yan Wang ${ }^{\dagger 1}$, Zheng-tang Chen*1, Wen-lei Zhuo', Bo Zhu', Rong-xia Liao $^{2}$ and Shao-xiang Zhang*3
}

\author{
Address: ${ }^{1}$ Cancer Institute of People's Liberation Army, Xinqiao Hospital, Third Military Medical University, Chongqing, 400037, PR China, \\ ${ }^{2}$ Department of Biochemistry and Molecular Biology, Third Military Medical University, Chongqing, 400038, PR China and ${ }^{3}$ Department of \\ Anatomy, College of Medicine, Third Military Medical University, Chongqing, 400038, PR China \\ Email: Jian-guo Sun - sunjianguo1972@yahoo.com.cn; Yan Wang - yanwang2327@yahoo.com.cn; Zheng- \\ tang Chen* - zhengtangchen@yahoo.com.cn; Wen-lei Zhuo - zhuowenlei@163.com; Bo Zhu - oncology_bozhu@yahoo.com.cn; Rong- \\ xia Liao - liaorongxia06@yahoo.com.cn; Shao-xiang Zhang* - zhangcvh@yahoo.com.cn \\ * Corresponding authors †Equal contributors
}

Published: 16 February 2009

Journal of Experimental \& Clinical Cancer Research 2009, 28:21 doi:10.1 I86/1756-9966-28-2I

This article is available from: http://www.jeccr.com/content/28/I/2I

(C) 2009 Sun et al; licensee BioMed Central Ltd.

This is an Open Access article distributed under the terms of the Creative Commons Attribution License (http://creativecommons.org/licenses/by/2.0), which permits unrestricted use, distribution, and reproduction in any medium, provided the original work is properly cited.
Received: 21 October 2008

Accepted: 16 February 2009

\begin{abstract}
Background: Our aim was to detect lymphatic endothelial marker podoplanin, lymphatic vessel endothelial hyaluronan receptor-I (LYVE-I) and vascular endothelial growth factor receptor-3 (VEGFR)-3 and study the prognostic relevance of lymphangiogenesis in non-small cell lung cancer (NSCLC).

Materials: 82 paraffin-embedded tissues and 40 fresh frozen tissues from patients with NSCLC were studied. Tumor samples were immunostained for the lymphatic endothelial markers. Lymphangiogenesis was assessed by immunohistochemical double stains for Podoplanin and Ki-67. The prognostic relevance of lymphangiogenesis-related clinicopathological parameters in NSCLC was evaluated.
\end{abstract}

Results: We found that the number of podoplanin positive vessels was correlated positively with the number of LYVE-I positive vessels. Most of VEGFR-3 positive, few of LYVE-I positive and none of podoplanin positive vessels were blood vessels. Peritumoral lymphatic vessel density (ptLVD), pathologic stage, lymph node status, lymphatic vessel invasion (LVI), vascular endothelial growth factor-C (VEGF-C) expression and $\mathrm{Ki}-67$ index of the endothelium cells of the micro lymphatic vessels $(\mathrm{Ki} 67 \%)$ were associated significantly with a higher risk of tumor progress. ptLVD, pathologic stage, lymph-node metastasis and Ki67\% were independent prognostic parameters for overall survival.

Conclusion: Podoplanin positive ptLVD might play important roles in the lymphangiogenesis and progression of NSCLC. Patients with high podoplanin+ ptLVD have a poor prognosis.

\section{Background}

The major cause of death from malignant tumors including non-small cell lung cancer (NSCLC) is dissemination of the primary tumor, leading to formation of metastases. Spread to regional lymph nodes is often the first step of generalization. Thus, the presence of lymph node metas- 
tasis represents a major criterion for evaluating the prognosis of NSCLC patients. Tumor-associated lymphangiogenesis are considered as the main route for lymphatic metastasis. And lymphovascular invasion (LVI) of tumor cells is a prerequisite for the dissemination via the lymphatic system. However, Studies of lymphatic vessels and lymphogenic metastasis have been hampered by the lack of specific lymphatic markers. Recently several markers for normal and tumor-associated lymphatic vessels have provided tools for a detailed analysis of lymphangiogenesis in human lung cancers. These markers include vascular endothelial growth factor $\mathrm{C}$ and $\mathrm{D}$ (VEGF-C, VEGF-D) [1,2], vascular endothelial growth factor receptor-3 (VEGFR-3) [3-6], the lymphatic vessel endothelial hyaluronan receptor-1 (LYVE-1) [7] and glomerular podocyte membrane mucoprotein podoplanin [8]. Podoplanin had been speculated as a specific lymphatic endothelial marker in many solid tumors including breast cancer, cervical cancer, Ovarian Cancer and NSCLC $[9,10]$. However, there are some contradictory results between different studies and many problems to be clarified. For example, VEGFR-3 is expressed not only in lymphatic endothelium in normal adult tissue, but also in vascular endothelium in tumor tissue. Therefore, using VEGFR-3 as a marker of tumor lymph vessel may lead to loss of accuracy in lymphatic vessel density (LVD) counting [11]. LYVE-1 was thought to be restricted to lymphatic vessels [12]. However, LYVE-1 was also found in normal hepatic blood sinusoidal endothelial cells and macrophage $[13,14]$. The specificity of LYVE-1 for lymphatic endothelial cells (LECs) has been questioned by some investigators [15]. Futhermore, Padera [16] showed that approximately 10\% of LYVE-1 + vessels were indeed blood vessels, suggesting that LYVE-1 alone is not suitable for the detection of functional lymphatic vessels. Until recently, tumorologists have recognized podoplanin as the most specific marker for lymphatic endothelium. And a double immunostaining with the D2-40 and anti-Ki67 monoclonal antibody is used as the standard method for the assessment of lymphangiogenesis in solid tumors[17]. Thus, the aim of this study was to detect Lymphangiogenesis and find the relationship between clinicopathological parameters, such as LVD, lymph-node metastasis, VEGFC, LVI, pathological stage, and prognostic factor in NSCLC.

\section{Methods}

\section{Patients and tissues}

This retrospective study included 82 patients with NSCLC who underwent either lobectomy or pneumonectomy at Xinqiao Hospital between January 1995 and November 2004. All of these patients have complete clinical and pathological records. None of the patients received presurgical radio- or chemotherapy before operation. Followup was made to August 31, 2005, by phone call, letter inquiry and visiting census register agency. During the follow-up period, there were 35 patients still alive and 47 deaths. Patients who were lost to follow up or died for noncancer-related reasons were excluded. Pathological stage was reevaluated and determined with the present TNM classification as revised in WHO 2004 classification criteria. Formalin-fixed, paraffin-embedded NSCLC tissues were retrieved from the files of our pathology department. Tissue blocks containing a representative fraction of the tumor and the tumor-lung parenchyma interface were used. Operative tissues embedded with paraffin from the 82 patients with NSCLC. In addition, the fresh frozen operation tissues of 40 NSCLC patients from Xinqiao and Daping hospital were used for LYVE-1 immunohistochemistry and H\&E staining (LYVE-1 expression was only on the fresh frozen sections, not on paraffin sections). The study was approved by the Ethics Committee (Faculty of Medicine, Third Military Medical University).

\section{Immunostaining for LYVE-I, CD3 I, VEGFR-3, podoplanin, VEGF-C and Vessel Counts}

Immunohistochemical stainings were done on tissues fixed in 10\% neutral buffered formalin and embedded in paraffin. Paraffin sections $(5 \mu \mathrm{m})$ were dewaxed and rehydrated. For light microscopy, peroxidase was quenched with methanol and $3 \% \mathrm{H}_{2} \mathrm{O}_{2}$ for 15 minutes. Antigen retrieval was done in $0.1 \mathrm{~mol} / \mathrm{L}$ citrate buffer $(\mathrm{pH}=6)$ in an $800 \mathrm{~W}$ microwave for 15 minutes (the step was omitted in fresh frozen section staining). After washing in PBS, the following primary antibodies were used: rabbit polyclonal anti-human LYVE-1 (10 $\mu \mathrm{g} / \mathrm{ml}$, Angiobio Co, USA), rabbit monoclonal anti-human podoplanin (1:100, Angiobio Co, USA), mouse monoclonal anti-human CD31 (ready to use, Zhongshan, Beijing), rabbit polyclonal anti-human VEGFR-3, VEGF-C (ready to use, Zhongshan, Beijing). All primary and secondary IgGs were diluted in PBS. Isotypic controls were performed for monoclonal as well as use of non immune serum for polyclonal antibodies (same concentrations as the test antibodies). Determination of LVD (assessed by immunostaining for podoplanin, LYVE-1, VEGFR-3) and CD31 microvessel density (MVD) was performed as suggested by Weidner [18]. Briefly, the immunostained sections were first scanned at a low magnification $(40 \times)$, and the areas with the greatest number of microvessels (vessel "hot spots") were selected for further evaluation. The microvessel count was then determined by counting all immunostained vessels in five separate hot spots at a high magnification $(\times 200)$. The average number of LVD or MVD in the five selected vessel hot spots was then calculated. In immunostainings for CD31, podoplanin, LYVE1 and VEGFR-3, any positive cell clusters were considered as endothelial cells and countable microvessels. LVI was considered evident if at least one tumor cell cluster was clearly visible inside the podoplanin-stained vascular 
space [19]. Peritumoral lymphatic vessels were defined as LYVE-1/podoplanin/VEGFR-3-positive vessels within an area of $100 \mu \mathrm{m}$ from the tumor border. Intratumoral lymphatic vessels were defined as LYVE-1/podoplanin/ VEGFR-3-positive vessels located within the tumor mass and not confined by invagination of normal tissue [20].

\section{Double immunostaining with podoplanin and Ki-67}

Immunohistochemical double stains for Podoplanin and Ki67 were done on serial sections according to Van den Eynden's method [21]. Podoplanin and Ki-67 was stained by D2-40 and anti-Ki67 monoclonal antibody, respectively. (Angiobio \& Beijing Zhongshan Jinqiao Biotechnology Co., respectively) Histastain ${ }^{\mathrm{TM}}$-DS double immunostaining kit was purchased from Zymed. In brief, sections were first incubated with primary antibody, i.e. podoplanin (dilution 1:200), and biotinized secondary antibody, which was visualized with the Envision + dual link system (Dakocytomation, Carpinteria, CA, USA). A second primary antibody, i.e. Ki67 (dilution 1:100) was then applied and visualized with the Envision G/2 system/AP (Dakocytomation, Carpinteria, CA, USA). Micro lymphatic vessels were brownish yellow after staining, while the nucleus of the proliferating endothelium cells of the micro lymphatic vessels appeared red. Ki-67 index of the endothelium cells of the micro lymphatic vessels (Ki67\%) was calculated according to Wulff et al [22].

\section{Statistical Analysis}

Correlations between podoplanin, VEGFR-3, LYVE-1 and the vessel numbers as continuous variables were used to assess CD31-positive vessel counts with the Spearman rank correlation test. Categorical data were compared by the $\chi^{2}$ or Fishers' exact probability test. Distribution was normal or with Mann Whitney U test if the sample distribution was asymmetrical. The relationship between lymph vessel variables and lymph node status was analyzed by one-way ANOVA, followed by the NeumanKeuls test. Overall survival intervals were determined as the time period from initial diagnosis to the time of death. Overall survival analyses were done using the KaplanMeier method. The comparison between survival functions for different strata was assessed with the log-rank statistic. Multivariate analysis of prognostic factors was done using Cox's regression model. Differences were considered significant when $P \leq 0.05$. All statistical analyses were done using the statistical package spss13.0.

\section{Results}

\section{CD3I, VEGFR-3, LYVE-I, VEGF-C Expression in NSCLC}

Numerous intratumoral and peritumoral vessels could be observed in each NSCLC tumor irrespective of histologic grade and pathologic stage. CD31 was positive in endothelial cell plasma in micro vessels, appeared yellow granular. Micro vessels of tumor tissues were mainly located at intra-tumor and peritumoral area. However, large blood vessels with muscular coat were also positive stained for CD31 (Fig. 1a). VEGFR-3 showed an expression similar to CD31. VEGFR-3 positive vessels included not only dilated and irregular thin-walled lymphatic vessels, but also blood vessels containing erythrocytes and large blood vessels with smooth muscle (Fig. 1b). LYVE-1 was positively stained in endothelial cell plasma and plasma membrane in micro vessels, appeared yellow granular (Fig. 1c). However, few LYVE-1 positive vessels were large blood vessels with smooth muscle, and tumor embolus were observed in their muscular layer and lumen (Fig. 1d). VEGF-C positive substance in tumor tissue was yellow fine granular, mainly located in tumor cell plasma. Positive cells were dispersed, limited locally or in small patches (Fig. 1e). In the para-tumor normal bronchia, VEGF-C expression was dispersed in columnar epithelium cells (Fig. 1f).

\section{Podoplanin Expression in NSCLC}

Podoplanin expression was mainly present in thin-walled (lymphatic) structures. Podoplanin was positive in endothelial cell plasma in thin-walled lymph vessel, appeared yellow granular. Podoplanin-positive lymph vessels were commonly located around the tumor or at the tumor boundary, mainly located at adenocarcinoma interstitium, peri-tumor area of squamous cell carcinoma and interstitium of large cell lung cancer (Fig. 2a, b, c). 4 cases of squamous cell carcinoma also demonstrated podoplanin expression in cancer cell plasma (data not shown). Moreover, we cut serial sections of lung cancer tissue, and stained them with podoplanin, CD31 and VEGFR-3, respectively. The red arrow in Fig. 2d indicates podoplanin-negative blood vessels. Black arrow in Fig. $2 \mathrm{~d}$ indicates podoplanin-positive lymph vessel. While in Fig. $2 \mathrm{e}$ and $2 \mathrm{f}$, the same region was positively stained for CD31 and VEGFR-3, indicating that VEGFR-3 was also a marker of blood vessels.

\section{Correlation analysis of podoplanin, LYVE-I, VEGFR-3 and CD3I}

In 82 paraffin-embedded NSCLC tissues, the mean number of podoplanin ${ }^{+}$vessels was $21.5 \pm 8.4$ (range $7.4-$ 43.6). The mean number of CD31 and VEGFR-3 ${ }^{+}$vessels was $51.4 \pm 11.1$ (range 30.0-77.2) and 30.2 \pm 16.8 (range 0-46.6), respectively. No substantial association was found between the number of podoplanin ${ }^{+}$vessels and CD $31^{+}$or VEGFR-3 ${ }^{+}$vessels (the Spearman rank correlation coefficient $r=-0.171, P=0.124 ; r=0.003, P=0.979$, respectively). In contrast, high counts of VEGFR-3+ vessels were strongly associated with high CD31+ vessel counts $(r$ $=0.331, P=0.002$ ), which showed most VEGFR-3+vessels were microvalscular vessels not lymphatic vessels. 

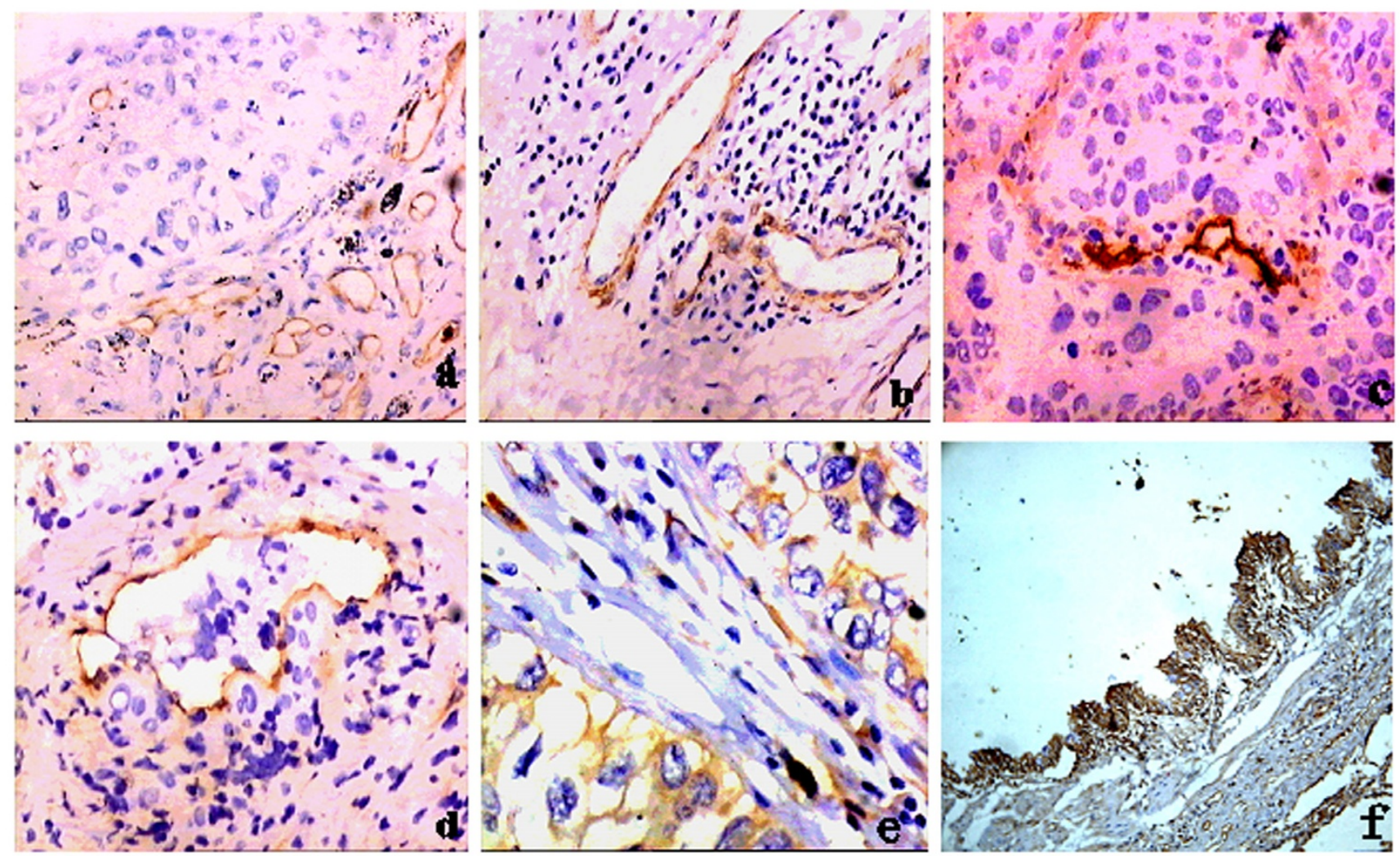

Figure I

Immunohistochemical analysis of different markers.

In addition, in 40 frozen NSCLC tissues, the mean number of LYVE-1+ vessels was $19.9 \pm 9.0$ (range 5.248.0). The mean number of CD31 and podoplanin ${ }^{+}$vessels was $52.3 \pm 10.9$ (range 34.4-71.2) and $22.1 \pm 8.1$ (range 6.6-44.6), respectively. No substantial association was found between the number of CD31+ vessels and LYVE-1 or podoplanin ${ }^{+}$vessels $(r=0.009, P=0.957 ; r=$ $0.059, P=0.717$, respectively). In contrast, high counts of LYVE-1+ vessels were strongly associated with high podoplanin $^{+}$vessel counts $(r=0.525, P=0.001)$. With the results of morphology above mentioned, LYVE-1+ vessels were most lymphatic vessels, but few of them were micro vessels.

\section{VEGF-C expression in NSCLC tissue and its relation to lymph node metastasis}

Carcinoma VEGF-C expression was classified either as positive ( $n=61, \geq 10 \%$ of the carcinoma cells expressed VEGF-C) or negative ( $n=21$, absent expression or expression in $<10 \%$ of the carcinoma cells). Among the 82 NSCLC tissues, 61 were VEGF-C positive, 21 were negative, indicating a positive expression rate of $74.4 \%$ (61/ $82)$. The positive expression rate was significantly higher in the lymph node positive group $(93.2 \%, 41 / 44)$ than in the lymph node negative group $(52.6 \%, 20 / 38)(P=$ 0.000) (Fig. 3a). ptLVD of patients was significantly higher in the VEGF-C positive group than in the VEGF-C negative group $(23.1 \pm 8.5$ vs $15.6 \pm 4.2, P=0.000)$. However, intratumoral lymphatic vessel density (itLVD) values of the two groups showed no significant difference (10.7 \pm 5.3 vs $10.4 \pm 4.7, P=0.820$ ) (Fig. $3 \mathrm{~b}$ ). According to spearman correlation analysis, VEGF-C expression was positively correlated with ptLVD and lymph node metastasis $(r=0.367, P=0.001 ; r=0.463, P=0.000)$. Moreover, ptLVD was also positively correlated with lymph node metastasis $(r=0.354, P=0.001)$.

\section{Associations of Lymphangiogenesis with Clinicopathological Parameters in NSCLC}

Double immunostaining with podoplanin and Ki-67 was performed for lymphogenesis analysis (Figure 4). Micro lymphatic vessels were brownish yellow after staining, while the nucleus of the proliferating endothelium cells of the micro lymphatic vessels appeared brownish red (indicated by the red arrow). Cancer embolus was detected in lymphatic vessels(Figure 4a). 

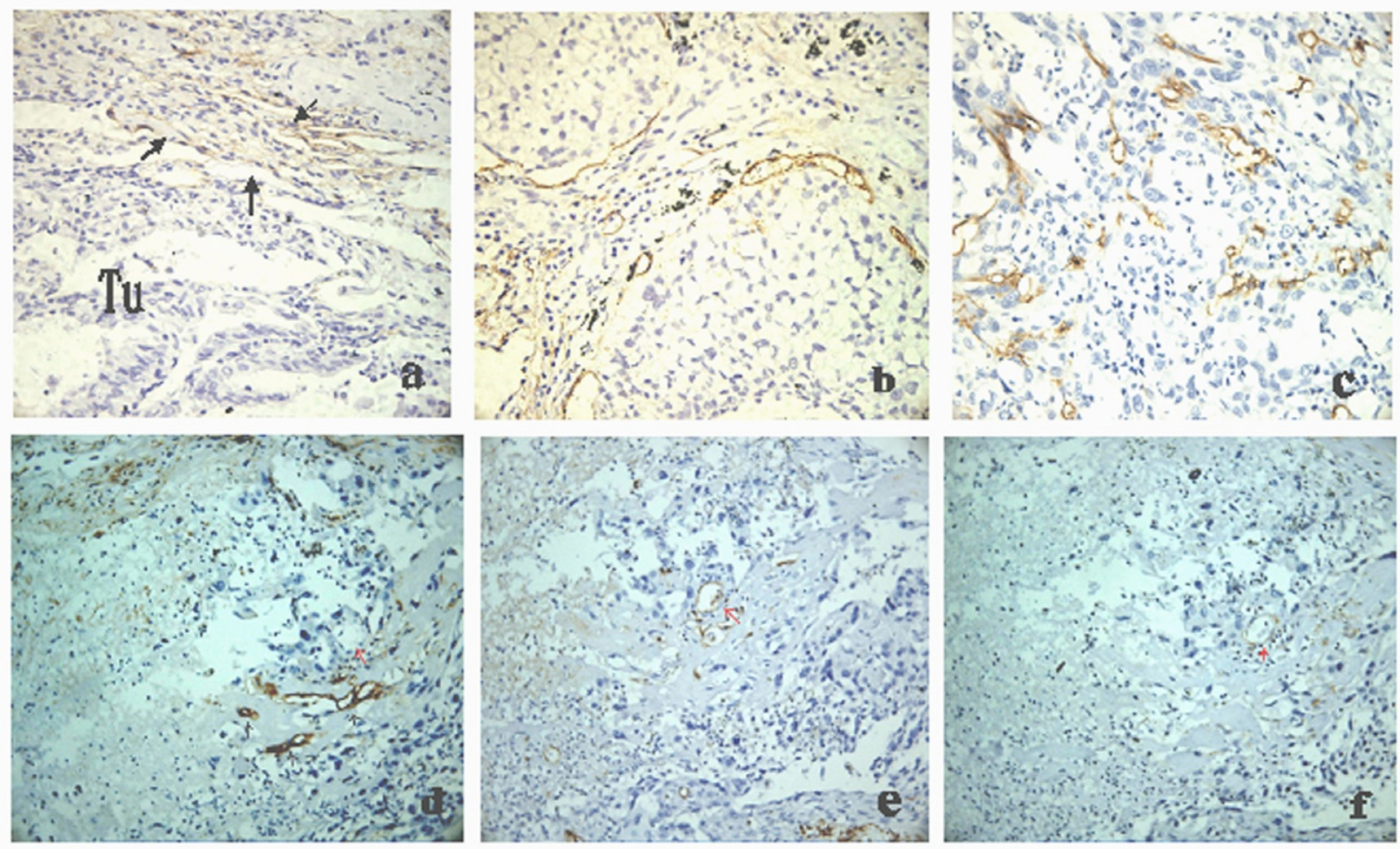

Figure 2

Immunostaining for podoplanin in nsclc tissues.

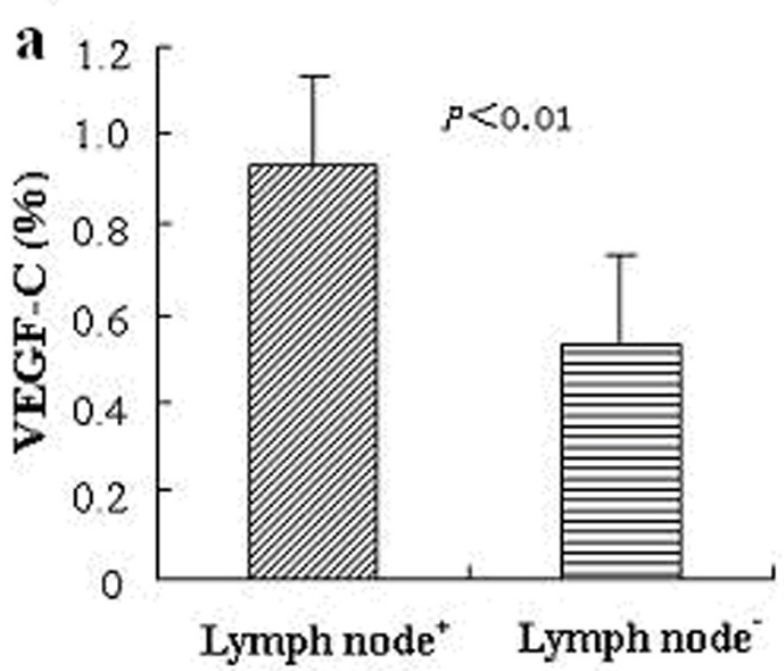

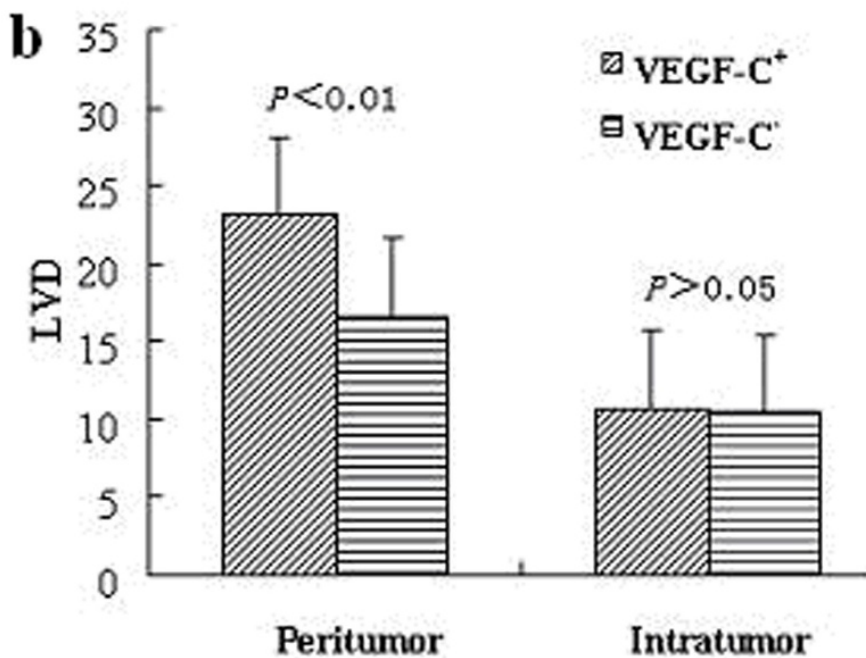

Figure 3

Prognosis analysis of vegf-c expression. 

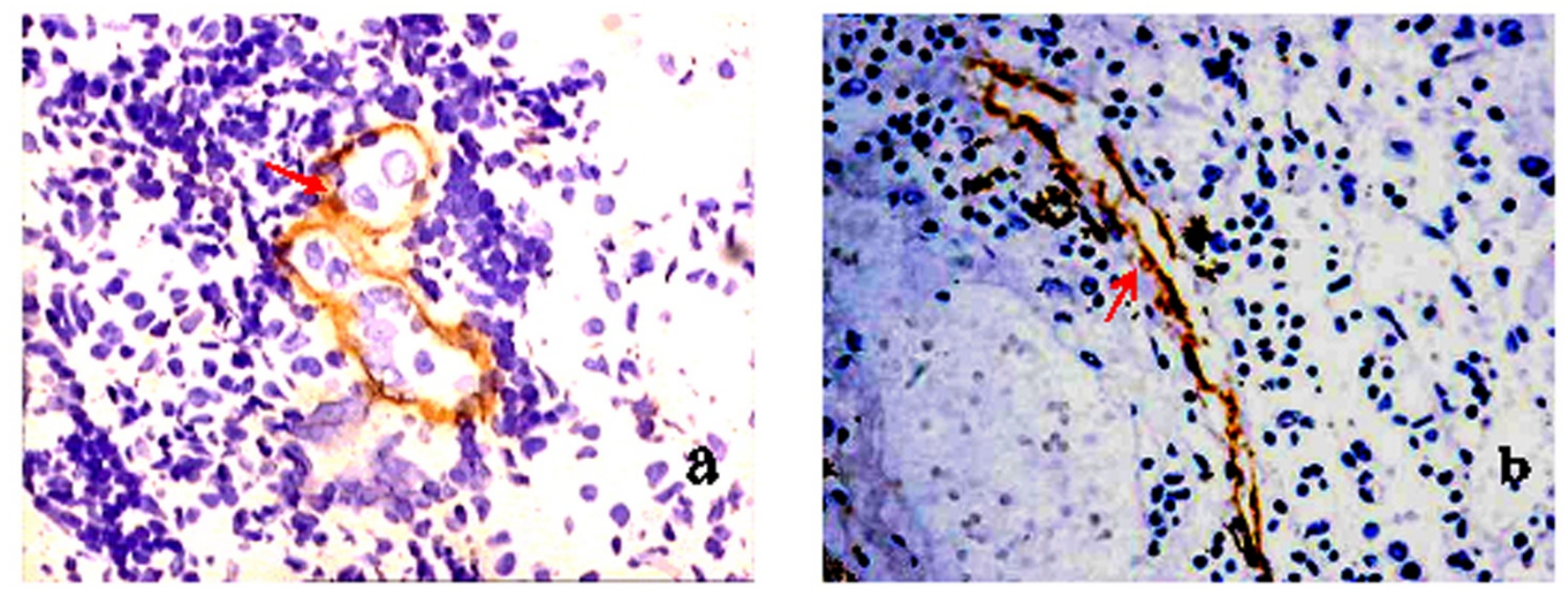

Figure 4

Double immunostaining with podoplanin and ki-67.

Table I: Association of LVD and LVI with other clinicopathological parameters

\begin{tabular}{|c|c|c|c|c|c|c|c|}
\hline \multicolumn{2}{|c|}{ Clinicopathological Parameters } & \multirow{2}{*}{$\begin{array}{c}\text { Cases } \\
42\end{array}$} & \multirow{2}{*}{$\begin{array}{c}\text { ptLVD } \\
22.1 \pm 8.9\end{array}$} & \multirow{2}{*}{$\begin{array}{c}\text { itLVD } \\
10.1 \pm 5.1\end{array}$} & \multirow{2}{*}{$\begin{array}{l}\text { LVI } \\
(-/+) \\
17 / 25\end{array}$} & \multirow{2}{*}{$\begin{array}{c}\begin{array}{c}\text { Mean Survival Time } \\
(x \pm s)\end{array} \\
1567 \pm 138\end{array}$} & \multirow{2}{*}{$\begin{array}{c}\text { median survival time } \\
1658\end{array}$} \\
\hline Age $(y)^{*}$ & $\geqq 55$ & & & & & & \\
\hline & $<55$ & 40 & $20.8 \pm 7.9$ & $11.2 \pm 5.1$ & $21 / 19$ & $|856 \pm 24|$ & 1864 \\
\hline \multirow[t]{2}{*}{ Gender } & male & 63 & $21.0 \pm 7.9$ & $11.2 \pm 4.9$ & $27 / 36$ & $1795 \pm 183$ & 1658 \\
\hline & female & 19 & $23.2 \pm 9.9$ & $8.7 \pm 5.5$ & $1 \mathrm{I} / 8$ & $1578 \pm 214$ & 1577 \\
\hline \multirow[t]{3}{*}{ Histologic type } & Squamous cell & 31 & $19.7 \pm 6.4$ & $9.6 \pm 4.6$ & $14 / 17$ & $1664 \pm 189$ & 1972 \\
\hline & Adenocarcinoma & 41 & $22.4 \pm 9.5$ & $11.0 \pm 5.1$ & $20 / 21$ & $1815 \pm 231$ & 1337 \\
\hline & Large cell & 10 & $23.4 \pm 9.1$ & $12.4 \pm 6.1$ & $4 / 6$ & $1134 \pm 156$ & 1118 \\
\hline \multirow[t]{2}{*}{ Tumor differentiation } & Well-moderate & 44 & $21.3 \pm 8.6$ & $10.5 \pm 5.4$ & $20 / 24$ & $2085 \pm 220$ & 1900 \\
\hline & Poor & 38 & $21.7 \pm 8.3$ & $10.8 \pm 5.0$ & $18 / 20$ & $1325 \pm 154$ & 1118 \\
\hline \multirow[t]{2}{*}{ Pathologic $N$ stage } & $\mathrm{NI}-2$ & 44 & $24.2 \pm 8.9 \#$ & $10.6 \pm 5.4$ & $15 / 29 \#$ & $943 \pm 107 \#$ & 674 \\
\hline & No & 38 & $18.4 \pm 6.7$ & $1 \mathrm{I} .7 \pm 4.7$ & $22 / 16$ & $2725 \pm 213$ & 2545 \\
\hline \multirow[t]{2}{*}{ Pathologic T stage } & $\mathrm{T} 2-3$ & 51 & $20.9 \pm 8.6$ & $11.4 \pm 5.2$ & $21 / 30$ & $1449 \pm 149$ & 1223 \\
\hline & TI & 31 & $22.5 \pm 8.0$ & $9.5 \pm 4.8$ & $17 / 14$ & $1875 \pm 172$ & 1775 \\
\hline \multirow[t]{2}{*}{ BVI } & $\mathrm{BVI+}$ & 39 & $23.2 \pm 9.8$ & $9.8 \pm 4.3$ & $21 / 18$ & $132 \mid \pm 146$ & 1117 \\
\hline & BVI- & 43 & $19.9 \pm 6.6$ & $11.3 \pm 5.7$ & $|2 / 2|$ & $2083 \pm 230$ & 2031 \\
\hline \multirow[t]{2}{*}{ ptLVD* } & $\operatorname{High}(\geq 19.9)$ & 41 & 1 & $12.7 \pm 5.6$ & $13 / 28 \#$ & $117 \mid \pm 153^{\#}$ & 772 \\
\hline & $\operatorname{Low}(<19.9)$ & 41 & 1 & $12.2 \pm 4.9$ & $25 / 16$ & $2378 \pm 224$ & 2057 \\
\hline \multirow[t]{2}{*}{ itLVD* } & $\mathrm{High}(\geq 10.2)$ & 46 & $22.9 \pm 7.4$ & I & $23 / 23$ & $1749 \pm 229$ & 1577 \\
\hline & $\operatorname{Low}(<10.2)$ & 36 & $23.3 \pm 6.7$ & 1 & $|5 / 2|$ & $1675 \pm 162$ & 1658 \\
\hline \multirow[t]{2}{*}{ LVI } & $\mathrm{LVI+}$ & 46 & $24.0 \pm 9.3^{\#}$ & $10.9 \pm 5.4$ & I & $1212 \pm 125^{\#}$ & 1006 \\
\hline & LVI- & 36 & $18.2 \pm 5.8$ & $10.3 \pm 4.7$ & I & $2433 \pm 245$ & 2123 \\
\hline \multirow[t]{2}{*}{ Pathologic stage } & $|+| \mid$ & 48 & $19.4 \pm 7.6 \#$ & $10.8 \pm 4.9$ & $26 / 22^{\#}$ & $2501 \pm 202^{\#}$ & 2115 \\
\hline & III+IV & 34 & $24.5 \pm 8.7$ & $10.4 \pm 5.4$ & $11 / 23$ & $800 \pm 105$ & 621 \\
\hline \multirow[t]{2}{*}{ VEGF-C } & Positive & 61 & $23.1 \pm 8.5^{\#}$ & $10.6 \pm 5.0$ & $24 / 37 \#$ & $1519 \pm 173 \#$ & 1117 \\
\hline & Negative & 21 & $16.9 \pm 6.0$ & $10.7 \pm 5.7$ & $14 / 7$ & $2232 \pm 194$ & 1981 \\
\hline \multirow[t]{2}{*}{ Ki67/\%* } & $\operatorname{High}(\geq 3.56)$ & 50 & $24.2 \pm 9.2^{\#}$ & $12.9 \pm 4.4$ & $21 / 29 \#$ & $1322 \pm 135^{\#}$ & 1109 \\
\hline & $\operatorname{Low}(<3.56)$ & 32 & $17.2 \pm 4.8$ & $13.3 \pm 5.0$ & $21 / 11$ & $2431 \pm 235$ & 2024 \\
\hline
\end{tabular}

*Cutoff value is median value. \#Correlation is statistically significant.

(BVI: Blood vessel invasion, LVI: lymphatic vessel invasion, ptLVD: peritumoral lymphatic vessel density, itLVD: intratumoral lymphatic vessel density, $\mathrm{Ki} 67 / \%$ : $\mathrm{Ki}-67$ index of the endothelium cells of the micro lymphatic vessels) 
The clinic significance was studied by analyzing the peritumoral and intratumoral lymphangiogenesis, various pathological parameters and follow-up data in 82 cases of NSCLC (Table 1). We divided LVD into high LVD Group and low LVD Group according to median. Then the differences was analyzed one by one between ptLVD and itLVD in Age, Gender, Histologic type, Tumor differentiation, Pathologic N stage, Pathologic T stage, Blood vessel invasion (BVI), LVI, Pathologic stage, VEGF-C expression and Ki67\%. The mean itLVD was 10.2. No difference was found in any factors between Group high itLVD $(\mathrm{n}=46)$ and Group low itLVD $(\mathrm{n}=36)(P>0.05$ for all analyses). But ptLVD was different. The median ptLVD was 19.9. No difference was found in LVI, age, gender, the primary tumor size, histologic grade and histologic type between Group high ptLVD $(\mathrm{n}=41)$ and Group low ptLVD ( $\mathrm{n}=$ 41) $(P>0.05)$. However, high ptLVD Group showed a significant increase than low ptLVD Group in several other clinicopathological parameters, such as lymph node metastasis, LVI, pathologic stage, VEGF-C and Ki67\%. Namely, ptLVD was higher in stage III a patients than in stage I and II $(P<0.01)$, in LVI positive than in LVI negative $(P=0.004)$, in lymph node metastasis than in lymph node negative $(P<0.01)$, in VEGF-C positive than in VEGF-C negative $(P<0.01)$ in high Ki67\% than in low Ki67\%. ptLVD were associated significantly with a higher risk for developing LVI and lymph-node metastasis $(P<$ $0.01)$.

\section{Associations of LVI with Clinicopathological Parameters}

Likewise, the relationship was analyzed between LVI and Age, Gender, Histologic type, Tumor differentiation, Pathologic $\mathrm{N}$ stage, Pathologic $\mathrm{T}$ stage, Blood vessel invasion, LVI, Pathologic stage, VEGF-C expression and Ki67\% (Table 1). Data showed that LVI were significantly associated with lymph-node metastasis, ptLVD, Pathologic stage, VEGF-C expression and Ki67\% $(P<0.01)$, but not with itLVD, Pathologic T stage and Blood vessel invasion (BVI).

\section{Lymphangiogenesis and Prognostic factor in NSCLC}

The overall survival rate (OS) was $49.3 \%$ in 82 NSCLC cases in five years. The median observation time was 1291 days (ranging from 103 to 3680 days). The Kaplan-Meier survival rate curve was showed in Fig 5a. Among it, five year survival rate was $33.5 \%$ in $\mathrm{LVI}^{+}$patients, and $70.0 \%$ in LVI- ones. By log-rank test, it was significantly different in survival rate curve in Fig $5 b(P=0.0002)$. Five year survival rate was $31.0 \%$ in high ptLVD patients, and $67.6 \%$ in low ptLVD ones, showing significant difference in survival rate curve (Fig 5c) $(P=0.0001)$. Five year survival rate was $50.0 \%$ in high itLVD patients, and $48.7 \%$ in low itLVD ones, showing no significant difference in survival rate curve (Fig $5 \mathrm{~d})(P=0.7045)$. In univariate survival analysis, intramural LVD $(P=0.719)$, as well as the patient's age, gender and other clinical and histopathologic parameters had no influence on OS in our collective ( $P>0.05$ for all analyses). A significant difference in OS was found between patients with high and those with low podoplanin ${ }^{+}$ptLVD (groups cut off by LVD median) $(P=$ $0.0001)$, with and without LVI $(P=0.0002)$, lymph node status $(P=0.0000)$, pathologic stage $(P=0.0000)$, VEGFC expression $(P=0.0054)$ and $\mathrm{Ki} 67 \%(P=0.0001)$. A multivariate analysis of these individuals was performed using the Cox regression Model. ptLVD, pathologic stage, lymph-node metastasis and Ki67\% were independent prognostic parameters for overall survival $(P=0.028)$ (Table 2). Podoplanin positive ptLVD might play important roles in the lymphangiogenesis and progression of NSCLC. Patients with high podoplanin+ ptLVD have a poor prognosis.

\section{Discussion}

There are many reports about tumor angiogenesis and poor prognosis in NSCLC. For example, Carcinoembryonic antigen-related cell adhesion molecule 1 (CEACAM1 ) has recently been reported to be implicated in cancer development and progression. The elevated CEACAM-1 expression and increased MVD, was an unfavorable prognosis in NSCLC [23]. It has also been reported that high CD34 ${ }^{+}$MVD and tumour vessel invasion are more closely

Table 2: Multivariate analysis of various prognostic factors in patients with NSCLC

\begin{tabular}{|c|c|c|c|c|}
\hline \multirow[b]{2}{*}{ Prognostic factor } & \multirow{2}{*}{$\begin{array}{c}\text { Univariate } \\
P \text { value }\end{array}$} & \multicolumn{3}{|c|}{ Multivariate } \\
\hline & & $\beta$ & P value & Relative Risk (95\%) Cl) \\
\hline ptLVD (high/low) & 0.0001 & 0.828 & 0.003 & $2.288(1.182-4.428)$ \\
\hline Pathologic stage $(|+| I / I I I+\mid V)$ & 0.0000 & 1.310 & 0.003 & $3.708(1.58 I-8.694)$ \\
\hline Pathologic $\mathrm{N}$ stage $\left(\mathrm{N}_{0} / \mathrm{N}_{2-3}\right)$ & 0.0000 & 1.218 & 0.010 & $3.382(1.344-8.511)$ \\
\hline $\operatorname{LVI}(-/+)$ & 0.0002 & 0.714 & 0.052 & $2.041(0.993-4.196)$ \\
\hline VEGF-C $(-/+)$ & 0.0054 & -0.365 & 0.490 & $0.694(0.246-1.958)$ \\
\hline $\mathrm{Ki} 67 \%$ & 0.0012 & 0.726 & 0.032 & $2.067(1.026-4.161)$ \\
\hline
\end{tabular}

(LVI: lymphatic vessel invasion, ptLVD: peritumoral lymphatic vessel density, Ki67/\%: Ki-67 index of the endothelium cells of the micro lymphatic vessels) 

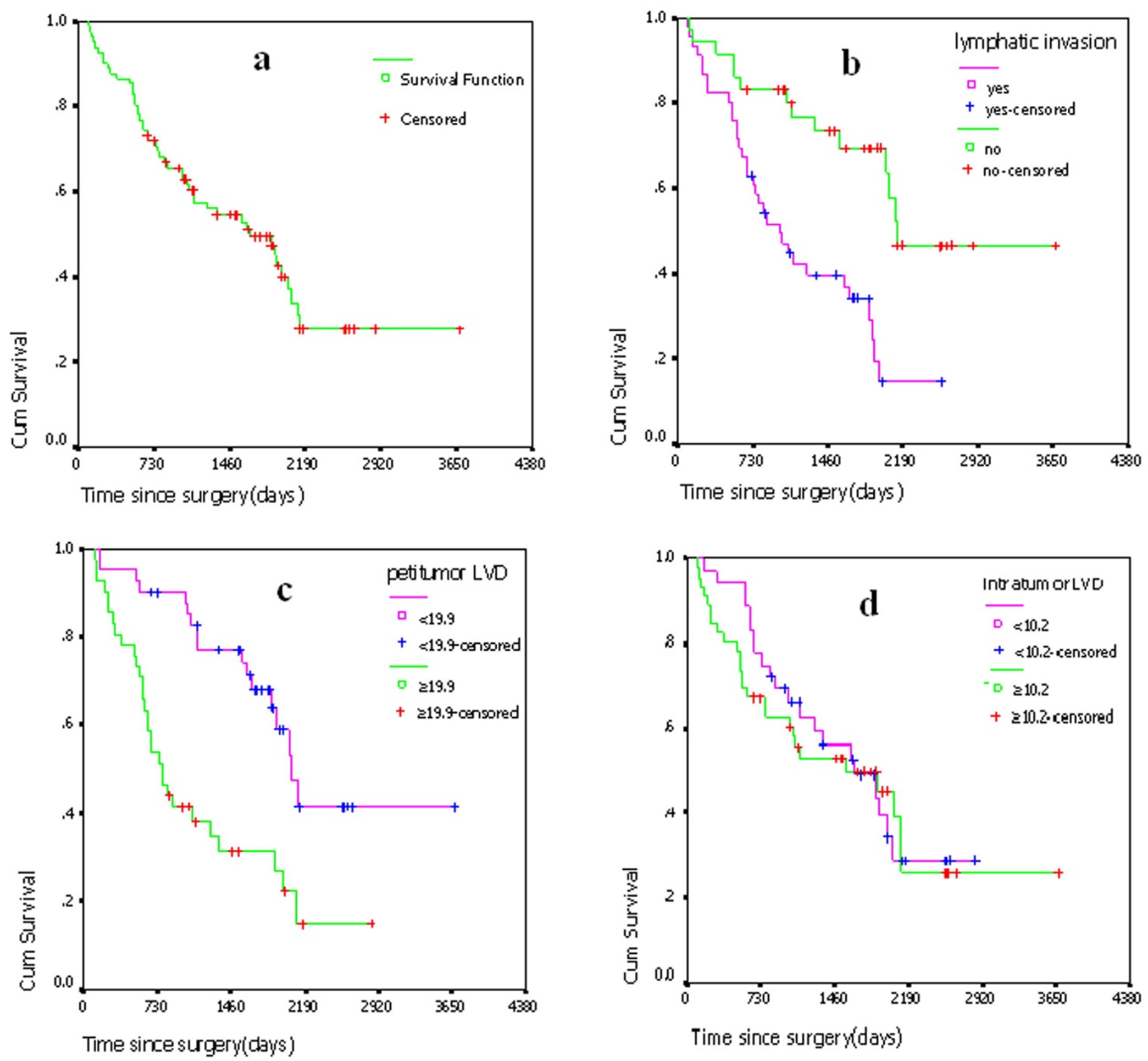

Figure 5

Survival analysis of clinicopathological parameters.

related to poor survival than the other neoangiogenetic factors in stage IB-IIA NSCLC [24]. In recent years, with the identification of lymphatic endothelial growth factorC (VEGF-C), VEGF-D and lymphatic endothelial markers including LYVE-1, VEGFR-3 and podoplanin, lymphangiogenesis has become one of the highlights in the field of metastasis in NSCLC. Active lymphangiogenesis is ongoing within sentinel lymph node (SLN) from NSCLC patients, even before metastasis. This lymphangiogenesis may be promoted by upregulation of VEGF121, which may in turn act in part through induction of VEGF-C [25]. Kadota [26] also showed that lymphangiogenesis, specifi- cally Micro-LVD was independently associated with poor prognosis of NSCLC patients. However, these researches can not indicate which LVD status was associated with prognosis of NSCLC patients. What is more, a Meta analysis has been finished [27]. 17 centers provided data for 3200 patients, 2719 of which were included in the analysis. For microvessel density counts obtained by the Chalkley method, the HR for death per extra microvessel was $1.05(95 \%$ CI 1.01-1.09, $P=0.03)$ when analyzed as a continuous variable. For microvessel density counts obtained by the all vessels method, the HR for death per ten extra microvessels was $1.03(0.97-1.09, P=0.3)$ when 
analyzed as a continuous variable. Microvessel density does not seem to be a prognostic factor in patients with non-metastatic surgically treated NSCLC. These conclusions contradict each other. Therefore, the methodology used to assess prognostic factors should be assessed carefully.

Positive correlation was found between the number of podoplanin positive vessels and the number of LYVE-1 positive vessels, while counts of VEGFR-3 positive vessels were correlated with CD31 positive vessel counts. Most of VEGFR-3 vessels, few of LYVE-1 and none of podoplanin positive vessels were blood vessels by observation of light microscope. The results were in accordance with Petri Bono's [28]. In specimens investigated in our study podoplanin expression was restricted to thin-walled lymph vessels with a single endothelial layer. Blood vessels containing red blood cells remained unstained. Podoplanin+ lymph vessels were almost peritumoral, not intratumoral. Lymph vessels could not form in the tumor because of low expression of lymphatic vessel growth factor and high expression of lymph vessel inhibitor factor in the tumor. Furthermore, high interstitial pressure in the tumor was caused with an increase size of lesions [29]. Our research also shows that podoplanin+ ${ }^{\text {ptLVD }}$ is associated with lymphatic metastasis, Pathologic stage and Ki67\%, and not with histologic type or Tumor differentiation. We presumed that high density of lymph vessels could increase cancer cells to contact with, and invade into lymph vessels, promote lymphatic metastasis and tumor progress. So, podoplanin ${ }^{+}$ptLVD is an independent prognostic parameter indeed. Patients with high podoplanin $^{+}$ptLVD have a poor prognosis. The result is consistent with the previous research. Saijo [30] showed the recurrence-free survival (RFS) time of patients with high Lymphatic permeation (ly 2) was significantly shorter than that of no Lymphatic permeation (ly 0$)$ patients $(P<$ $0.0001)$, and low Lymphatic permeation(ly 1$)$ patients $(P$ $=0.0028)$. A significant difference in RFS time was also observed between the ly 0 patients and the ly 1 patients $(P$ $=0.0025)$. RFS time of the ly 0 patients was significantly longer than that of the ly 1 plus ly 2 patients $(P<0.0001)$. Saijo only studied Lymphatic permeation (ly) in Lymphangiogenesis and prognosis of patients with NSCLC. Our study further shows that podoplanin ${ }^{+}$ptLVD not itLVD is the prognostic parameter. Podoplanin ${ }^{+}$ptLVD could also be useful to be a new antitumor target. However, these observations are based only on retrospective analysis of a small case series and further evaluation with a larger number of cases is necessary.

\section{Conclusion}

Podoplanin is the most specific lymphatic endothelial marker. ptLVD and lymph-node metastasis might play important roles in the onset and progression of NSCLC.

\section{Competing interests}

The authors declare that they have no competing interests.

\section{Authors' contributions}

IS conceived of the study, and participated in its design and drafted the manuscript. YW participated in the study design and collected the tissues and carried out the immunoassays. WZ and BZ participated in the immunoassays and performed the statistical analysis. RL helped with the statistical analysis and manuscript drafting. ZC and SZ conceived of the study, and participated in its design and coordination and helped to draft the manuscript.

\section{Acknowledgements}

This work was supported by grant from National Natural Science Foundation of China (to Zheng-tang Chen) (NO.3037I 586). It was also sponsored by Key project, Chongqing Natural Science Foundation (to Zheng-tang Chen) (NO. CSTC, 2005BA5006), and Scientific Research Foundation for Returned Overseas Chinese Scholars, Third Military Medical University (to Zheng-tang Chen) (NO. XG20036I).

\section{References}

I. Ogawa E, Takenaka K, Yanagihara K, Kurozumi M, Manabe T, Wada $H$, Tanaka $F$ : Clinical significance of VEGF-C status in tumour cells and stromal macrophages in non-small cell lung cancer patients. Br J Cancer 2004, 9 I (3):498-503.

2. Baldwin ME, Halford MM, Roufail S, Williams RA, Hibbs ML, Grail D, Kubo H, Stacker SA, Achen MG: Vascular endothelial growth factor $D$ is dispensable for development of the lymphatic system. Mol Cell Biol 2005, 25(6):244I-2449.

3. Arinaga M, Noguchi T, Takeno S, Chujo M, Miura T, Uchida Y: Clinical significance of vascular endothelial growth factor $C$ and vascular endothelial growth factor receptor 3 in patients with non small cell lung carcinoma. Cancer 2003, 97(2):457-464.

4. Saintigny P, Kambouchner M, Ly M, Gomes N, Sainte-Catherine O, Vassy R, Czernichow S, Letoumelin P, Breau JL, Bernaudin JF, Kraemer $M$ : Vascular endothelial growth factor- $C$ and its receptor VEGFR-3 in non-small-cell lung cancer: concurrent expression in cancer cells from primary tumour and metastatic lymph node. Lung Cancer 2007, 58(2):205-213.

5. Kojima H, Shijubo N, Yamada G, Ichimiya S, Abe S, Satoh M, Sato N Clinical significance of vascular endothelial growth factor-C and vascular endothelial growth factor receptor 3 in patients with TI lung adenocarcinoma. Cancer 2005, 104(8): I668-1677.

6. Berghmans T, Meert AP, Martin B, Ninane V, Sculier JP: Prognostic role of epidermal growth factor receptor in stage III nonsmall cell lung cancer. Eur Respir J 2005, 25(2):329-335.

7. Banerji S, Ni J, Wang SX, Clasper S, Su J, Tammi R, Jones M, Jackson DG: LYVE-I, a new homologue of the CD44 glycoprotein, is a lymph-specific receptor for hyaluronan. J Cell Biol 1999, I44(4):789-80I.

8. Breiteneder-Geleff S, Soleiman A, Horvat R, Amann G, Kowalski H, Kerjaschki D: Podoplanin a specific marker for lymphaticendothelium expressed in angiosarcoma. Verh Dtsch Ges Pathol 1999, 83:270-275.

9. Schoppmann SF, Birner P, Studer P, Breiteneder-Geleff S: Lymphatic microvessel density and lymphovascular invasion assessed by anti-podoplanin immunostaining in human breast cancer. Anticancer Res 200I, 2 I(4A):235I-2355.

10. Renyi-Vamos F, Tovari J, Fillinger J, Timar J, Paku S, Kenessey I, Ostoros G, Agocs L, Soltesz I, Dome B: Lymphangiogenesis correlates with lymph node metastasis, prognosis, and angiogenic phenotype in human non-small cell lung cancer. Clin Cancer Res 2005, II (20):7344-7353.

II. Partanen TA, Alitalo K, Miettinen M: Lack of lymphatic vascular specificity of vascular endothelial growth factor receptor 3 in I 85 vascular tumors. Cancer 1999, 86(I I):2406-24I2. 
12. Koukourakis MI, Giatromanolaki A, Sivridis E, Simopoulos C, Gatter KC, Harris AL, Jackson DG: LYVE-I immunohistochemical assessment of lymphangiogenesis in endometrial and lung cancer. J Clin Pathol 2005, 58(2):202-206.

13. Mouta Carreira C, Nasser SM, di Tomaso E, Padera TP, Boucher Y, Tomarev SI, Jain RK: LYVE-I is not restricted to the lymph vessels: expression in normal liver blood sinusoids and downregulation in human liver cancer and cirrhosis. Cancer Res 200I, 6 I (22):8079-8084.

14. Jackson DG: Biology of the lymphatic marker LYVE-I andapplications in research into lymphatic trafficking and lymphangiogenesis. APMIS 2004, II 2(7-8):526-538.

15. Schacht V, Dadras SS, Johnson LA, Jackson DG, Hong YK, Detmar M: Up-regulation of the lymphatic marker podoplanin, a mucintype transmembrane glycoprotein, in human squamous cel carcinomas and germ cell tumors. Am J Pathol 2005, 166(3):9|3-92|.

16. Padera TP, Kadambi A, di Tomaso E, Carreira CM, Brown EB, Boucher Y, Choi NC, Mathisen D, Wain J, Mark EJ, Munn LL, Jain RK: Lymphatic metastasis in the absence of functional intratumor lymphatics. Science 2002, 296(5574): | 883-1886.

17. Auwera I Van der, Cao Y, Tille JC, Pepper MS, Jackson DG, Fox SB, Harris AL, Dirix LY, Vermeulen PB: First international consensus on the methodology of lymphangiogenesis quantification in solid human tumours. Br J Cancer 2006, 95( I 2): 16 I I-1625.

18. Weidner N: Tumor angiogenesis: review of currentapplications in tumor prognostication. Semin Diagn Pathol 1993, I0(2):302-3।3.

19. Heimburg S, Oehler MK, Papadopoulos T, Caffier H, Kristen P, Dietl J: Prognostic relevance of the endothelial marker CD 34 in ovarian cancer. Anticancer Res 1999, I 9(4A):2527-2529.

20. Dadras SS, Lange-Asschenfeldt B, Velasco P, Nguyen L, Vora A, Muzikansky A, Jahnke K, Hauschild A, Hirakawa S, Mihm MC, Detmar M: Tumor lymphangiogenesis predicts melanoma metastasis to sentinel lymph nodes. Mod Pathol 2005, I8(9): I232- 1242.

21. Eynden GG Van den, Vandenberghe MK, van Dam PJ, Colpaert CG, vanDam P, Dirix LY, Vermeulen PB, Van Marck EA: Increasedsentinel lymph node lymphangiogenesis is associated with nonsentinelaxillary lymph node involvement in breast cancer patients with apositive sentinel node. Clin Cancer Res 2007 I3(I 8 Pt I):539|-5397.

22. Wulff C, Dickson SE, Duncan WC, Fraser HM: Angiogenesis in the human corpus luteum: simulated early pregnancy by HCG treatment is associated with both angiogenesis and vessel stabilization. Hum Reprod 200 I, I6( I 2):2515-2524.

23. Dango S, Sienel W, Schreiber M, Stremmel C, Kirschbaum A, Pantel $\mathrm{K}$, Passlick B: Elevated expression of carcinoembryonic antigen-related cell adhesion molecule I (CEACAM-I) is associated with increased angiogenic potential in non-small-cell lung cancer. Lung Cancer 2008, 60(3):426-433.

24. Mineo TC, Ambrogi V, Baldi A, Rabitti C, Bollero P, Vincenzi B, Tonini G: Prognostic impact of VEGF, CD3I, CD34, and CDI05 expression and tumour vessel invasion after radical surgery for IB-IIA non-small cell lung cancer. J Clin Pathol 2004, 57(6):59|-597.

25. Kawai H, Minamiya Y, Ito M, Saito H, Ogawa J: VEGFI 2 I promotes lymphangiogenesis in the sentinel lymph nodes of non-small cell lung carcinoma patients. Lung Cancer 2008, 59(I):4 I-47.

26. Kadota K, Huang CL, Liu D, Ueno M, Kushida Y, Haba R, Yokomise $\mathrm{H}$ : The clinical significance of lymphangiogenesis and angiogenesis in non-small cell lung cancer patients. Eur J Cancer 2008, 44(7): 1057-1067.

27. Trivella M, Pezzella F, Pastorino U, Harris AL, Altman DG, Prognosis In Lung Cancer (PILC) Collaborative Study Group: Microvessel density as a prognostic factor in non-small-cell lung carcinoma: a meta-analysis of individual patient data. Lancet Oncol 2007, 8(6):488-499.

28. Bono P, Wasenius VM, Heikkilä P, Lundin J, Jackson DG, Joensuu $H$ : High LYVE-I-positive lymphatic vessel numbers are associated with poor outcome in breast cancer. Clin Cancer Res 2004 I0(2I):7144-7|49.

29. Vleugel MM, Bos R, Groep P van der, Greijer AE, Shvarts A, Stel HV, Wall $E$ van der, van Diest PJ: Lack of lymphangiogenesis during breast carcinogenesis. J Clin Pathol 2004, 57(7):746-75I.

30. Saijo T, Ishii G, Ochiai A, Hasebe T, Yoshida J, Nishimura M, Nagai K: Evaluation of extratumoral lymphatic permeation in non- small cell lung cancer as a means of predicting outcome. Lung Cancer 2007, 55(1):6I-66.
Publish with Bio Med Central and every scientist can read your work free of charge

"BioMed Central will be the most significant development for disseminating the results of biomedical research in our lifetime. "

Sir Paul Nurse, Cancer Research UK

Your research papers will be:

- available free of charge to the entire biomedical community

- peer reviewed and published immediately upon acceptance

- cited in PubMed and archived on PubMed Central

- yours - you keep the copyright 